\title{
Trombosis venosa profunda como debut de leucemia aguda
}

\author{
Joaquín Ferreira Bacciarini*
}

\section{Resumen}

La relación existente entre trombosis y cáncer es ampliamente conocida. Sin embargo, este vínculo es frecuentemente subestimado en las neoplasias hematológicas, hecho que debe mantener alerta al médico clínico. La trombosis venosa profunda puede constituir la manifestación inicial de una leucemia aguda o precederla en el diagnóstico. La búsqueda minuciosa de esplenomegalia y linfadenopatías en el examen clínico, así como una mirada atenta al recuento completo de células sanguíneas (hemograma completo) son esenciales para no omitir el diagnóstico, con implicancias pronósticas y terapéuticas directas. Se presenta el caso clínico de una paciente con trombosis venosa profunda en el miembro inferior izquierdo como debut de una leucemia aguda.

Palabras clave: TROMBOSIS VENOSA PROFUNDA

LEUCEMIA AGUDA

RECUENTO COMPLETO DE CÉLULAS SANGUIINEAS

Key words: DEEP VEIN THROMBOSIS

ACUTE LEUKEMIA

COMPLETE BLOOD COUNT

\section{Introducción}

La enfermedad tromboembólica venosa (ETEV) constituye una patología frecuente en los servicios de emergencia y medicina interna, que genera alta morbimortalidad y costos en salud. La trombosis venosa profunda (TVP) y el tromboembolismo pulmonar representan sus formas clásicas de presentación clínica. Desde el punto de vista etiológico, la ETEV puede asociarse a diversas condiciones predisponentes, entre ellas el cáncer ${ }^{(1)}$. La relación entre trombosis y cáncer se tiene muy presente en el caso de los tumores sólidos. Por el contrario, en las neoplasias hematológicas este vínculo es frecuentemente subestimado y queda a la sombra de las complicaciones hemorrágicas, hecho que debe mantener alerta al médico clínico ${ }^{(2)}$. En diversos estudios se ha establecido que los pacientes con linfoma, mieloma múltiple y leucemia aguda tienen un riesgo trombótico aumentado, particularmente en el momento del diagnóstico y durante la etapa de quimioterapia ${ }^{(3)}$. A continuación se reporta el caso clínico de una paciente con TVP en el miembro inferior izquierdo como debut de una leucemia aguda, en el cual el recuento completo de células sanguíneas (o hemograma completo) constituyó la brújula en el camino diagnóstico.

\section{Caso clínico}

Se presenta el caso de una paciente de sexo femenino de 38 años de edad, con antecedentes personales de obesidad e hipertensión arterial en tratamiento con telmisartán, sin trombofilia previa conocida. En su historia gineco-obstétrica, la paciente presentaba el antecedente de seis embarazos, cuatro partos vaginales, una cesárea, un aborto espontáneo, un óbito fetal, y negaba el uso de anticonceptivos orales. No presentaba historia familiar de trombofilia ni de enfermedad oncológica.

\footnotetext{
* Internista de Clínica Médica "1", Hospital Maciel. Ex Jefe de Residentes de Medicina Interna, Hospital Maciel.

Correspondencia: Hospital Maciel, 25 de Mayo 174. Montevideo, Uruguay. Correo electrónico: ferreira.joaquin85@gmail.com El autor declara no tener conflictos de interés.

Recibido: 26/5/17

Aprobado: $10 / 12 / 17$
} 


\begin{tabular}{|c|c|c|}
\hline Parámetro & Resultados & Valores de referencia \\
\hline $\begin{array}{l}\text { Hematíes } \\
\left(\text { millones } / \mathrm{mm}^{3} \text { ) }\right.\end{array}$ & 3,38 & $4,20-5,00$ \\
\hline Hemoglobina (g/dl) & 9,6 & $12,0-15,0$ \\
\hline Hematocrito & 30,6 & $36,0-47,0$ \\
\hline VCM (fL) & 90,5 & $80,0-93,0$ \\
\hline CHCM (g/dl) & 31,4 & $32,0-36,0$ \\
\hline $\operatorname{ADE}(\%)$ & 16,5 & $11,6-14,8$ \\
\hline Leucocitos (mil/mm³) & 10,6 & $4,0-10,0$ \\
\hline Plaquetas $\left(\mathrm{mil} / \mathrm{mm}^{3}\right)$ & 150 & $150-400$ \\
\hline $\operatorname{TP}(\%)$ & 76,6 & $70,0-120,0$ \\
\hline TTPA (seg) & 35,8 & $26,0-36,0$ \\
\hline INR & 1,2 & $0,8-1,2$ \\
\hline Fibrinógeno (mg/dl) & 692 & $200-400$ \\
\hline Dímeros D (ng/ml) & 600 & $0-500$ \\
\hline \multicolumn{3}{|c|}{$\begin{array}{l}\text { VCM: volumen corpuscular medio; CHCM: concentración de he- } \\
\text { moglobina corpuscular media; ADE: ancho de distribución eritro- } \\
\text { citaria; TP: tiempo de protrombina; TTPA: tiempo de tromboplas- } \\
\text { tina parcial activada. }\end{array}$} \\
\hline
\end{tabular}

La paciente fue derivada desde un centro de atención primaria al servicio de emergencia del Hospital Maciel por edema de aparición repentina, localizado a nivel de pierna y muslo izquierdos, de tres días de evolución, que se acompañaba de dolor y rubor local. No existía historia previa de traumatismo o reposo prolongado, y la paciente negaba la presencia de disnea, dolor torácico o fiebre.

A su llegada a emergencia la paciente se encontraba lúcida, normotensa, en apirexia, con saturación de oxígeno basal de $96 \%$. Presentaba frecuencia respiratoria de 14 respiraciones/min y frecuencia cardíaca de 100 latidos/min, con buena perfusión periférica. Del examen físico general destacaba la presencia de obesidad (con un índice de masa corporal de 33) y palidez cutáneo-mucosa. La auscultación cardiovascular y pleuropulmonar eran normales. El examen abdominal mostraba un abdomen globuloso, blando e indoloro a la palpación profunda. A nivel de los miembros inferiores destacaba la presencia de edema asimétrico localizado en pierna y muslo izquierdos, rubor local con empastamiento y dolor a la palpación. No se encontraron lesiones cutáneas de puerta de entrada ni cordones varicosos visibles.
Tabla 2. Recuento total leucocitario y fórmula leucocitaria.

\begin{tabular}{lccc}
\hline $\begin{array}{l}\text { Tipo de } \\
\text { leucocito }\end{array}$ & $\begin{array}{c}\text { Valor absoluto } \\
\left(\mathrm{mil}^{\mathrm{mm}} \mathrm{mm}^{3}\right)\end{array}$ & $\begin{array}{c}\text { Porcentaje } \\
(\%)\end{array}$ & Valores de referencia \\
\hline Neutrófilos & 1,11 & 10,5 & $2,50-7,00-50,0-70,0$ \\
Linfocitos & 3,23 & 30,5 & $1,00-4,00-20,0-40,0$ \\
Monocitos & 5,77 & 54,5 & $0,05-1,00-5,0-10,0$ \\
Eosinófilos & 0,08 & 0,8 & $0,00-0,50-1,0-5,0$ \\
Basófilos & 0,02 & 0,2 & $0,00-0,10-0,0-1,0$ \\
\hline & & & \\
\hline
\end{tabular}

Los resultados de las pruebas sanguíneas complementarias se exponen en la tabla 1. De estos resultados destacaba la presencia de anemia normocítica y normocrómica, recuento plaquetario en el límite inferior de la normalidad e hiperfibrinogenemia. Los dímeros D se encontraban elevados. Las pruebas de coagulación básicas no mostraban alteraciones.

Se sospechó el diagnóstico de TVP dada la presencia de edema asimétrico en el miembro inferior izquierdo, obesidad (factor de riesgo para trombosis venosa) y dímeros D elevados. La ecografía Doppler confirmó una TVP ilíaco-femoral en etapa aguda. La vena poplítea y los ejes venosos de la pierna se encontraban permeables. Con estos hallazgos se inició tratamiento anticoagulante con heparinas de bajo peso molecular.

En la evolución se revisó la historia clínica y las pruebas complementarias realizadas. Se observó un recuento total de monocitos igual a $5.770 / \mathrm{mm}^{3}$, que representaba el 54,5\% del recuento leucocitario, asociado a neutropenia de $1100 / \mathrm{mm}^{3}$. En la tabla 2 se resumen los valores del recuento total de leucocitos y la fórmula leucocitaria.

Posteriormente, guiados por las alteraciones encontradas en el hemograma, se repitió el examen físico haciendo énfasis en una detallada exploración abdominal y linfoganglionar. Se constató la presencia de esplenomegalia grado 2, indolora. No se encontraron linfadenopatías.

En resumen, se trataba de una paciente con diagnóstico confirmado de TVP ilíaco-femoral izquierda, que presentaba anemia moderada, normocítica y normocrómica, inversión de la fórmula leucocitaria (con neutropenia), recuento plaquetario en el límite inferior de la normalidad y esplenomegalia. En función de las alteraciones hematológicas, sugestivas de una posible insuficiencia medular e infiltración extramedular, se sospechó la asociación del evento trombótico con una leucemia 
aguda. Con este diagnóstico presuntivo, se solicitó interconsulta con hematólogo. Se realizó un aspirado medular que confirmó un $80 \%$ de blastos mieloides en la médula ósea. El estudio inmunofenotípico por citometría de flujo demostró que la población blástica presentaba características fenotípicas sugestivas de una leucemia mieloide con mínima diferenciación. Los estudios citogenético, molecular y de hibridación in situ con fluorescencia no demostraron anomalías cromosómicas.

Seguidamente, con el diagnóstico confirmado de leucemia aguda mieloide, se inició quimioterapia de inducción con daunorrubicina y citarabina. En la evolución, la paciente desarrolló aplasia medular con trombocitopenia severa, y presentó genitorragia con repercusión hematimétrica; esta complicación hemorrágica obligó a suspender la anticoagulación y a considerar la colocación de un filtro cava. Posteriormente, la paciente desarrolló shock séptico refractario al tratamiento y falleció a los 13 días de haberse iniciado la quimioterapia.

\section{Discusión y comentarios}

La incidencia de ETEV en pacientes con neoplasias hematológicas puede ser similar a la observada en pacientes con tumores sólidos ${ }^{(4)}$. Un metaanálisis reciente incluyó 72 estudios prospectivos y 9.061 pacientes con leucemia. Dicho estudio determinó una tasa de incidencia de tromboembolismo venoso correspondiente a $6 \%$ para pacientes con leucemia aguda mieloide (LAM), y a otro 6\% para pacientes adultos con leucemia aguda linfoide (LAL) ${ }^{(5)}$. Asimismo, en un estudio de cohortes que incluyó alrededor de 8.000 pacientes con leucemia aguda, la incidencia acumulada total de ETEV a dos años fue de 5,2\% en pacientes con LAM y de $4,5 \%$ en pacientes con $\mathrm{LAL}^{(6)}$. Estas cifras son comparables a las reportadas en series de pacientes con cáncer de colon, esófago y riñón ${ }^{(6)}$. Con respecto a los diferentes subtipos de leucemia aguda, la mayor incidencia de trombosis ha sido reportada en la variante promielocítica ${ }^{(2)}$.

Las manifestaciones trombóticas ocurren particularmente en los primeros tres meses luego del diagnóstico de leucemia aguda, cuando los pacientes reciben un tratamiento intensivo ${ }^{(6)}$. Sin embargo, la ETEV puede representar el debut de la enfermedad hematológica, como sucedió en el caso analizado, o incluso preceder al diagnóstico en un período variable.

La patogenia de la ETEV relacionada con las neoplasias hematológicas es compleja y multifactorial, y está en estrecha relación con la enfermedad de base o su tratamiento $^{(3)}$. Algunos de los mecanismos involucrados incluyen la activación de la coagulación por medio de sustancias procoagulantes, la afectación de las vías fi- brinolíticas y la presencia de alteraciones a nivel del endotelio vascular ${ }^{(7)}$. Otros factores coadyuvantes para el desarrollo de ETEV incluyen la inmovilización, la cirugía, las infecciones, el uso de catéteres venosos centrales o la presencia de estados de hipercoagulabilidad hereditarios o adquiridos ${ }^{(3)}$. En relación con este último punto, la historia obstétrica de la paciente analizada podía sugerir la presencia de una trombofilia subyacente asociada. Sin embargo, no se obtuvo registro de estudios realizados previamente dirigidos a confirmar dicha posibilidad.

Los pacientes con leucemia aguda presentan un alto riesgo de hemorragia en relación al desarrollo de trombocitopenia por la propia enfermedad, o secundaria a la quimioterapia utilizada para su tratamiento ${ }^{(8)}$. En el caso analizado, la paciente desarrolló trombocitopenia profunda (secundaria a aplasia medular) y asoció genitorragia severa. Esta complicación hemorrágica demuestra que la terapia anticoagulante constituye un verdadero desafío en caso de confirmarse la ETEV asociada a leucemia aguda. Al igual que en otros tipos de cáncer, el tratamiento anticoagulante se basa en el uso de heparinas de bajo peso molecular, por su mejor perfil de seguridad en estos pacientes ${ }^{(8)}$. La entidad del sangrado obligó a suspender el tratamiento anticoagulante en esta paciente. No se logró colocar un filtro cava porque la paciente falleció de manera precoz a causa de una complicación infecciosa; pese a ello, no se registraron manifestaciones clínicas que orientaran a progresión del trombo o embolia pulmonar.

En el presente caso, una mirada con detenimiento al hemograma completo constituyó la pista clave para el diagnóstico precoz de leucemia aguda. La neutropenia (recuento de neutrófilos $<1500 / \mathrm{mm}^{3}$ ) se presenta entre el $30 \%$ y el $50 \%$ de los enfermos con LAM $^{(9)}$. La monocitosis (definida como el recuento de monocitos $>$ $800 / \mathrm{mm}^{3}$ ) puede verse en presencia de enfermedades granulomatosas (por ejemplo, tuberculosis o sarcoidosis) o algunos trastornos inflamatorios (por ejemplo, enfermedad inflamatoria intestinal), pero también puede ser un indicador de enfermedad hematológica maligna (leucemias agudas y crónicas, síndromes mielodisplási$\cos )^{(10)}$. La inversión de la fórmula leucocitaria nos condujo al reexamen clínico exhaustivo en busca de elementos orientadores a una hemopatía subyacente a la presentación del fenómeno trombótico. El diagnóstico definitivo se confirmó en colaboración con el hematólogo.

Cabe mencionar que en este caso el hallazgo de esplenomegalia fue omitido durante el examen físico inicial. La palpación abdominal tiene una buena especificidad para la detección de esplenomegalia (89\%-99\%), pero su sensibilidad es variable $(20 \%-70 \%)$ y depende 
no solo de la experiencia del examinador, sino también del tamaño del órgano ${ }^{(11)}$. Pueden ocurrir falsos negativos en presencia de, por ejemplo, obesidad, ascitis o ángulos costales estrechos $^{(11)}$. En esta paciente, la obesidad pudo ser la causa por la cual inicialmente se obvió la presencia de esplenomegalia. La esplenomegalia constituyó un dato semiológico fundamental para el proceso de razonamiento diagnóstico, hecho que subraya la importancia de un examen físico exhaustivo en los pacientes con manifestaciones clínicas de ETEV.

\section{Conclusiones}

Es importante recordar que los fenómenos trombóticos venosos pueden constituir la primera manifestación clínica de una leucemia aguda. Un examen físico minucioso y un correcto análisis e interpretación del hemograma completo pueden representar la clave para el médico clínico en el proceso diagnóstico.

\section{Agradecimientos}

Al Dr. Martín Elizondo, por sus aportes en la discusión del caso clínico.

\section{Abstract}

The relationship between thrombosis and cáncer is widely knkown. However, this relationship is often underestimated in hematologic neoplasms, what needs to be borne in mind my clinicians. Deep vein thrombosis (DVT) may constitute the initial manifestation of acute leukemia or precede its diagnosis. The meticulous search for splenomegaly and lymphadenopathies in the clinical examination, as well as the careful analysis of complete blood count are crucial for diagnostic purposes, and has direct prognostic and therapeutic implications. The study presents the clinical case of a patient with deep vein thrombosis in the left lower limb as a debut of acute leukemia.

\section{Resumo}

A relação entre trombose e câncer é muito conhecida. No entanto, este vínculo é frequentemente subestimado nas neoplasias hematológicas, o que deve ser um alerta para o médico clínico. A trombose venosa profunda pode ser a manifestação inicial de uma leucemia aguda ou precedê-la no diagnóstico. A investigação minuciosa de esplenomegalia e linfadenopatias no exame clínico, bem como uma leitura atenta do hemograma completo são essenciais para não omitir o diagnóstico que consequentes implicações diretas sobre o prognóstico e a terapêutica. Descreve-se um caso clínico de uma paciente com trombose venosa profunda no membro inferior esquerdo como inicio de uma leucemia aguda.

\section{Bibliografía}

1. Falanga A, Rickles FR. Pathophysiology of the thrombophilic state in the cancer patient. Semin Thromb Hemost 1999;25(2):173-82.

2. Oehadian A, Iqbal M, Sumantri R. Deep Vein Thrombosis in Acute Myelogenous Leukemia. Acta Med Indones 2009; 41(4):200-4.

3. Colombo R, Gallipoli P, Castelli R. Thrombosis and Hemostatic Abnormalities in Hematological Malignancies. Clin Lymphoma Myeloma Leuk 2014;14(6):441-50.

4. Guzmán-Uribe P, Vargas-Ruíz ÁG. Thrombosis in Leukemia: Incidence, Causes, and Practical Management. Curr Oncol Rep 2015;17(5):444.

5. Wu YY, Tang L, Wang MH. Leukemia and Risk of Venous Thromboembolism: A Meta-analysis and Systematic Review of 144 Studies Comprising 162,126 Patients. Sci Rep. 2017 Apr 26;7(1):1167.

6. Ku GH, White RH, Chew HK, et al. Venous thromboembolism in patients with acute leukemia: incidence, risk factors, and effect on survival. Blood 2009; 113:3911-3917.

7. De Stefano V, Sora F, Rossi E, et al. The risk of thrombosis in patients with acute leukemia: occurrence of thrombosis at diagnosis and during treatment. J Thromb Haemost 2005; 3 : 1985-92.

8. Falanga A, Marchetti M, Russo L. Venous thromboembolism in the hematologic malignancies. Curr Opin Oncol 2012;24(6):702-10.

9. Sans-Sabrafen J, Besses Raebel C, Vives Corrons JL. Hematología Clínica. $5^{\mathrm{a}}$ ed. Madrid: Elsevier, 2006; 409-415.

10. Argente HA, Alvarez ME. Semiología Médica: Fisiopatología, Semiotecnia y Propedéutica. Enseñanza-aprendizaje centrada en la persona. $2^{\mathrm{a}}$ ed. Buenos Aires: Médica Panamericana, 2013; 1123-27.

11. Salvatore M. Diagnóstico físico: Secretos. $2^{\mathrm{a}}$ ed. España: Elsevier, 2010; 467-72. 